\title{
A selective role for $\alpha 3$ subunit glycine receptors in inflammatory pain
}

\author{
Victoria L. Harvey' , Alex Caley ${ }^{2}$, Ulrike C. Müller ${ }^{3}$, Robert J. Harvey ${ }^{2}$ and Anthony H. Dickenson ${ }^{1 *}$ \\ 1 Neuroscience, Physiology \& Pharmacology, University College London, London, UK \\ 2 Department of Pharmacology, The School of Pharmacy, University of London, London, UK \\ ${ }^{3}$ Institute for Pharmacy and Molecular Biotechnology, University of Heidelberg, Heidelberg, Germany
}

\section{Edited by:}

Jean-Michel Rigo, Universiteit Hasselt, Belgium

\section{Reviewed by:}

Joe Lynch, University of Queensland, Australia

Jochen C. Meier, Max Delbrück Center for Molecular Medicine, Germany

${ }^{*}$ Correspondence:

Anthony H. Dickenson, Neuroscience, Physiology \& Pharmacology, University College London, Gower Street, London WC1E 6BT, UK

e-mail:anthony.dickenson@ucl.ac.uk
GlyR $\alpha 3$ has previously been found to play a critical role in pain hypersensitivity following spinal $\mathrm{PGE}_{2}$ injection, complete Freund's adjuvant (CFA) and zymosan induced peripheral inflammation. In this study, although all models displayed typical phenotypic behaviours, no significant differences were observed when comparing the pain behaviours of Glra3 $3^{--}$and wild-type littermates following the injection of capsaicin, carrageenan, kaolin/carrageenan or monosodium iodoacetate, models of rheumatoid and osteoarthritis, respectively. However, clear differences were observed following CFA injection $(p<0.01)$. No significant differences were observed in the pain behaviours of Glra3 $3^{-1}$ and wild-type littermates following experimentally induced neuropathic pain (partial sciatic nerve ligation). Similarly, Glra3 ${ }^{--}$and wild-type littermates displayed indistinguishable visceromotor responses to colorectal distension (a model of visceral pain) and in vivo spinal cord dorsal horn electrophysiology revealed no differences in responses to multimodal suprathreshold stimuli, intensities which equate to higher pain scores such as those reported in the clinic. These data suggest that apart from its clear role in CFA- and zymosan-induced pain sensitisation, hypersensitivity associated with other models of inflammation, neuropathy and visceral disturbances involves mechanisms other than the EP2 receptor - GlyR $\alpha 3$ pathway.

Keywords: glycine $\alpha 3$ subunit, prostaglandin $\mathrm{E}_{2}$ inflammation, visceral pain, arthritis, electrophysiology

\section{INTRODUCTION}

Despite recent developments in the understanding of pain mechanisms and analgesia, the relief of pain, particularly that which lasts over long periods, remains a principal concern to healthcare professionals. Chronic pain can be broadly categorised into three types: inflammatory, neuropathic and dysfunctional. Inflammatory pain, arising from tissue damage caused by trauma or infection, is associated with conditions such as arthritis. Neuropathic pain, arising from trauma to, or pathological changes in, the peripheral or central nervous systems can occur as a consequence of stroke, ischaemia, diabetes or nerve infection (e.g. HIV, shingles, etc.). The third type of pain is underpinned by neural dysfunction and is thought to be associated with conditions such as irritable bowel syndrome (IBS), fibromyalgia and migraine which manifest as non-localised diffuse pain unaccompanied by either inflammation or nerve damage.

Tissue damage precipitates the release of multifarious endogenous pro-inflammatory mediators at sites in both the periphery and the CNS (mainly at the level of the spinal cord). Peripheral release of these mediators, namely prostaglandin $\mathrm{E}_{2}\left(\mathrm{PGE}_{2}\right)$, leads to the sensitization of primary afferents, thereby lowering thresholds for neuronal activation, and increasing nociceptor responsivity. This increased peripheral activity contributes to spinal hypersensitivity (central sensitisation) resulting in increased responsivity to noxious stimulation termed hyperalgesia, and allodynia where normally nonnoxious stimuli such as cooling, gentle touch, movement and pressure are now perceived as being painful. Central prostanoid release can induce spinal disinhibition, relieving dorsal horn neurones from inhibitory control via spinal interneurones, thus facilitating the transmission of nociceptive information (Ahmadi et al., 2002; Reinold et al., 2005). One such mechanism of spinal disinhibition occurs through the postsynaptic activation of G-protein-coupled $\mathrm{EP}_{2}$ receptors, and subsequent protein kinase $\mathrm{A}$ induced inhibition of $\alpha 3$ subunit containing glycine receptors, present in the superficial laminae of the spinal cord (Harvey et al., 2004).

GlyR $\alpha 3$ subunit knockout mice ( $\mathrm{Glra}^{-/-}$) allow us to probe the function of this ligand-gated ion channel in the absence of any subtype-selective ligands. Previously, a role for GlyR $\alpha 3$ in inflammatory pain has been demonstrated in the CFA and zymosan A models of peripheral inflammation (Harvey et al., 2004). By contrast, GlyR $\alpha 3$ appears to have little or no role in neuropathic pain and the formalin test (Racz et al., 2005; Hösl et al., 2006). In this study, we have investigated the nociceptive phenotypes of GlyR $\alpha 3$ knockout mice in acute models of inflammation and clinically relevant models of persistent inflammatory, neuropathic and visceral pain. Moreover, we investigated the role of GlyR $\alpha 3$ in response to multimodal suprathreshold stimuli-evoking responses, which equate to higher pain scores experienced by human pain patients.

\section{MATERIALS AND METHODS ANIMALS}

Glra3 $^{--}$mice on a C57Bl/6 background were produced as described (Harvey et al., 2004). Glra3 ${ }^{-/}$mice and their wild-type littermates were group housed on a standard 12-h light/dark cycle with food and water available ad libitum except during behavioural testing. 
Experiments were performed on animals of at least 8 weeks of age. For all the experiments detailed in this study, the observer was blinded with respect to mouse genotype. All experiments were carried out in accordance with the UK Home Office Animals Scientific procedures Act (1986).

\section{INDUCTION AND BEHAVIOURAL ANALYSIS OF PAIN STATES}

Glra3 ${ }^{-1-}$ and wild-type littermates received either unilateral intraplantar injections of $1 \mathrm{mg} / \mathrm{ml}$ complete Freund's adjuvant (CFA, $20 \mu \mathrm{l}), 2 \%(\mathrm{w} / \mathrm{v})$ carrageenan $(20 \mu \mathrm{l})$ or $50 \mu \mathrm{g} / \mathrm{ml}$ capsaicin $(5 \mu \mathrm{l})$. Models of rheumatoid arthritis and osteoarthritis were induced by unilateral intra-articular injections of 3\% (w/v) kaolin/carrageenan $(\mathrm{K} / \mathrm{C} ; 15 \mu \mathrm{l})$, or $5 \mathrm{mg} / \mathrm{ml}$ monosodium iodoacetate (MIA, $10 \mu \mathrm{l}$; Harvey and Dickenson, 2009), respectively. Neuropathic (partial sciatic nerve ligation, PSNL) mice were prepared by unilateral tight ligation of $1 / 3$ to $1 / 2$ of the sciatic nerve using 7-0 silk under isofluorane/O $\mathrm{O}_{2}$ anaesthesia (Malmberg and Basbaum, 1998). Behavioural thermal hypersensitivity (thermal hyperalgesia) was assessed using the Hargreaves' test and the hot plate, and mechanical hypersensitivity (mechanical allodynia) was assessed using von Frey filaments as described (Harvey and Dickenson, 2009). Two baseline measurements were recorded prior to the induction of the pain models. Although both contralateral and ipsilateral limbs were tested, only ipsilateral data are shown for clarity since contralateral data were not significantly different from baseline following the induction of any pain model $(p>0.05)$. Capsaicin-induced acute spontaneous pain behaviours, measured as either biting, flinching, or licking, were measured for $3 \mathrm{~min}$.

\section{SPINAL CORD ELECTROPHYSIOLOGY}

Following a laminectomy (L3-L6) in vivo electrophysiology was performed in urethane anesthetised $(240 \mathrm{mg} / \mathrm{kg}) \mathrm{Glra3}^{-1-}$ and wild-type mice using parylene coated tungsten electrodes (A-M Systems, USA), as previously described (Harvey and Dickenson, 2009). Evoked neuronal responses were recorded from single wide dynamic range dorsal horn neurones receiving inputs from the hindpaw. Transcutaneous electrical stimuli consisted of a train of 16 electrical stimuli ( $2 \mathrm{~ms}$ wide pulses, $0.5 \mathrm{~Hz}, 3 \times \mathrm{C}$ fibre threshold), were delivered by means of pins inserted in to the hindpaw. Post stimulus time histograms were constructed and fibre responses were separated according to the following latencies: A (0-50 ms),
C (50-250 ms) and post discharge (250-800 ms). Input (i.e. non-potentiated response) is measured as the number of action potentials in response to the initial stimulus $\times 16$ (total number of stimuli). Wind up values, a measure of the temporal summation of neuronal activity in response to a train of stimuli, was calculated as the total number of action potentials evoked at the end of 16 stimuli minus the input. A wide range of natural stimuli including brush (dynamic mechanical) acetone (innocuous cold), and graded punctate mechanical and thermal stimuli applied using von Frey filaments and a water jet, respectively, were applied to the hindpaw for a period of $10 \mathrm{~s}$. Data were captured and analysed using a CED 1401 interface coupled to a Pentium computer running Spike 2 software (Cambridge Electronic Design).

\section{ACUTE VISCERAL NOCICEPTION}

Visceral nociception was assessed using a method adapted from Kamp et al. (2003). Under isofluorane anaesthesia (2.5\%, delivered in $\mathrm{O}_{2}$ ), an electromyographic (EMG) electrode (insulated copper wire, exposed at the ends and $1 \mathrm{~cm}$ centrally) was sewn into the external oblique muscle. A latex balloon $(20 \mathrm{~mm})$ attached to a pressure transducer via polyethylene tubing, with the distal $15 \mathrm{~mm}$ perforated using a 27-gauge needle to facilitate balloon inflation, was inserted intra-anally $(25 \mathrm{~mm})$, and secured by surgical tape to the base of the tail. The anaesthesia was gradually reduced to $\sim 1 \%$ isofluorane, and allowed to equilibrate for at least $30 \mathrm{~min}$, such that the animals did not display spontaneous movement but responded to cutaneous hindpaw stimulation. EMG responses were filtered, amplified and recorded in $10 \mathrm{~s}$ prior to and during colorectal distensions (10 s) of 10-60 $\mathrm{mmHg}$ applied at 4 min intervals. Two consecutive sets of CRD were recorded and averaged. Data are presented relative to the mean wild type maximum response (60 $\mathrm{mmHg})$.

\section{RESULTS}

\section{ACUTE NOCICEPTION}

Pain thresholds to punctate mechanical stimuli were measured in $\mathrm{Glra3}^{-/-}$mice and wild-type littermates using calibrated von Frey hairs, according to the up-down method (Chaplan et al., 1994). Paw withdrawal thresholds to mechanical stimuli were not significantly different between groups ( $p>0.05$; Figure 1A). Similarly, thermal thresholds assessed using the Hargreaves' test were indistinguishable
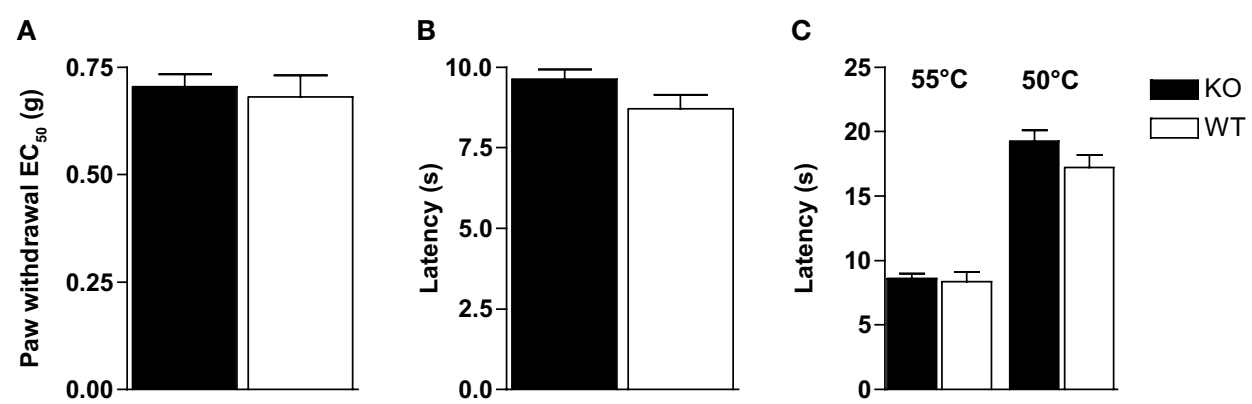

FIGURE 1 | GIra3 $^{-1-}$ mice show no differences in their acute pain behaviours. (A) Punctate mechanical stimulation using calibrated von Frey (Glra3 ${ }^{--}, n=16$; wild type, $n=10$ ). Thermal stimulation using either (B) the
Hargreaves' apparatus (Glra3 ${ }^{--}, n=30$; wild type, $n=19$ ) or (C) the hot plate at $50^{\circ} \mathrm{C}$ or $55^{\circ} \mathrm{C}\left(\mathrm{Glra3}^{-1}, n=12\right.$; wild type, $\left.n=12\right)$. Data are presented as mean \pm SEM. 
between groups ( $p>0.05$; Figure 1B). Thermal hypersensitivities were similar across genotypes when measured using the hot plate at either $50^{\circ} \mathrm{C}$ or $55^{\circ} \mathrm{C}(p>0.05$; Figure 1C). This absence of an acute phenotype in $\mathrm{Glra3}^{-1-}$ mice agrees with previous findings (Harvey et al., 2004).

Spinal cord electrophysiology was performed to assess the potential role of GlyR $\alpha 3$ in acute nociception in response to multimodal stimuli. Where behaviour lends information concerning threshold intensities, electrophysiology can be used to inform us about responses to suprathreshold stimuli. Single unit dorsal horn neuronal recordings were performed in $\mathrm{Glra3}^{-1-}(n=27)$ and wildtype littermates $(n=22)$. The mean depths from the surface of the spinal cord were not significantly different between groups and correspond to the deep laminae of the spinal cord $550 \pm 30 \mu \mathrm{m}$ and $459 \pm 38 \mu \mathrm{m}(p>0.05)$, respectively ensuring that the population of neurons was similar between the two groups, and hence direct comparisons were possible. Responses to electrical, brush, acetone, noxious cold and pinch (Table 1); as well as graded mechanical and thermal stimuli (Figures 2A,B) were indistinguishable between genotypes $(p>0.05)$.

Table 1 | Glra3 $^{-1}$ mice show no alteration in their neuronal responses to multimodal stimuli. Data are presented as mean \pm SEM for deep dorsal horn neurones recorded in G/ra3 ${ }^{--}(n=27)$ and wild-type mice $(n=22)$. AP represents the mean number of action potentials in response to either a peripheral electrical or 10-s natural stimulus.

\begin{tabular}{lcc}
\hline & Glra3- $^{- \text {- }}$ & Wild type \\
\hline A-fibre threshold (mA) & $0.05 \pm 0.08$ & $0.07 \pm 0.02$ \\
C-fibre threshold (mA) & $0.94 \pm 0.18$ & $0.92 \pm 0.16$ \\
A-fibre response (AP) & $105 \pm 10$ & $85 \pm 9$ \\
C-fibre response (AP) & $181 \pm 19$ & $143 \pm 18$ \\
Post discharge (AP) & $180 \pm 25$ & $155 \pm 21$ \\
Input (AP) & $216 \pm 28$ & $199 \pm 29$ \\
Wind-up (AP) & $186 \pm 31$ & $118 \pm 20$ \\
Brush (AP) & $67 \pm 22$ & $61 \pm 14$ \\
Pinch (AP) & $172 \pm 29$ & $154 \pm 25$ \\
Acetone (AP) & $14 \pm 5$ & $8 \pm 5$ \\
$1^{\circ} \mathrm{C}$ & $60 \pm 11$ & $48 \pm 20$
\end{tabular}

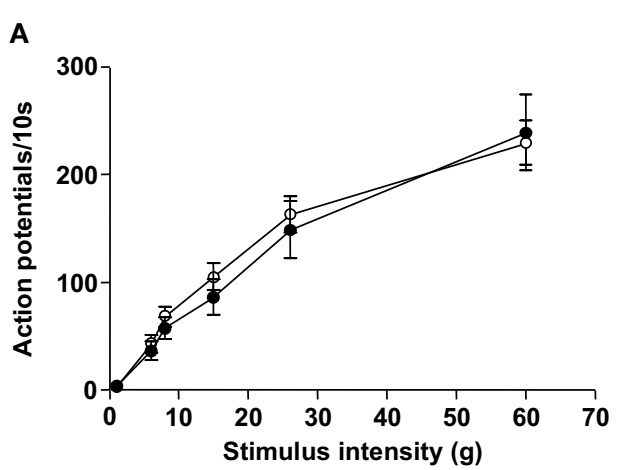

\section{INFLAMMATORY PAIN BEHAVIOUR}

Consistent with previously reported findings (Harvey et al., 2004) $\mathrm{Glra3}^{-1-}$ mice displayed showed no difference in the induction of thermal pain sensitisation following CFA $(20 \mu \mathrm{l})$ injection, but recovered much more quickly compared with wild-type littermate controls (Figure 3A). Paw withdrawal latencies to thermal stimulation using the Hargreaves' apparatus were significant versus contralateral responses for wild-type mice days 1-6 and days 1-3 for $\mathrm{Glra}^{-1-}$ mice $(p<0.001)$. Consistent with previous findings, Glra3 $^{--}$mice $(n=7)$ displayed significant differences in thermal hypersensitivity at 4, 5 and 6 days following CFA injection compared with wild-type littermates $(n=5, p<0.01)$.

Acute cutaneous neurogenic inflammation induced by intradermal injection of capsaicin $(5 \mu \mathrm{l} ; 50 \mu \mathrm{g} / \mathrm{ml})$ elicited spontaneous pain behaviour in both $\mathrm{Glra}^{-1-}$ and wild-type control mice (Figure 3B). No significant differences were observed between the type of nociceptive behaviour (i.e. licking, biting and flinching) or the duration of such responses $(p>0.05)$.

Intraplantar injection of carrageenan $(20 \mu \mathrm{l} ; 2 \% \mathrm{w} / \mathrm{v})$ elicited significant thermal and mechanical hypersensitivities in both $\mathrm{Glra3}^{-1-}$ and wild-type control mice at both acute (2-6 h), and sub-acute phases $(24 \mathrm{~h})\left(p<0.05-p<0.001 ; \mathrm{Glra}^{-1-}, n=5\right.$; wild type, $n=7$; Figures 3C,D). Statistical analysis revealed no significant difference in $\mathrm{Glra3}^{-1-}$ mice in either the magnitude or duration of thermal and mechanical hypersensitivities following carrageenan injection when compared with wild-type control littermates $(p>0.05)$.

\section{Clinically relevant models of inflammation}

Intra-articular injection of monosodium iodoacetate (MIA, $7 \mu \mathrm{l}$; $5 \mathrm{mg} / \mathrm{ml}$ ) to induce osteoarthritis (OA) evoked significant behavioural mechanical hypersensitivitiy in both $\mathrm{Glra}^{-/-}$and wildtype control mice when compared with contralateral controls $(n=6 ; p<0.001$; Figure 4A). Thermal hypersensitivity was not explored since this has been found to be absent in this model (Harvey and Dickenson, 2009). Similarly, a model of rheumatoid arthritis (kaolin/carrageenan; $15 \mu \mathrm{l}, 3 \% \mathrm{w} / \mathrm{v}$ ) evoked significant thermal hypersensitivity in both $\mathrm{Glra3}^{-1-}$ and wild-type control mice when compared with contralateral controls $(n=6$; $p<0.05$; Figure 4B). No significant differences were observed between genotypes.

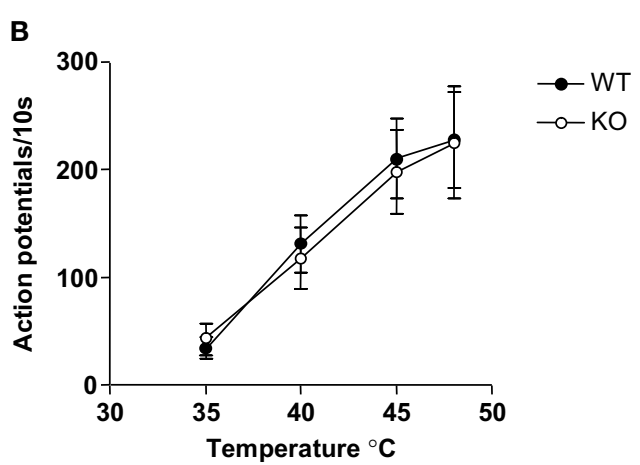

FIGURE 2 | GIra3--- mice show no differences in evoked dorsal horn neuronal responses. (A) Graded mechanical stimuli (von Frey); (B) graded thermal stimuli (water jet). Data are presented as mean \pm SEM (Glra3 ${ }^{-1}, n=27$; wild type, $\left.n=22\right)$. 

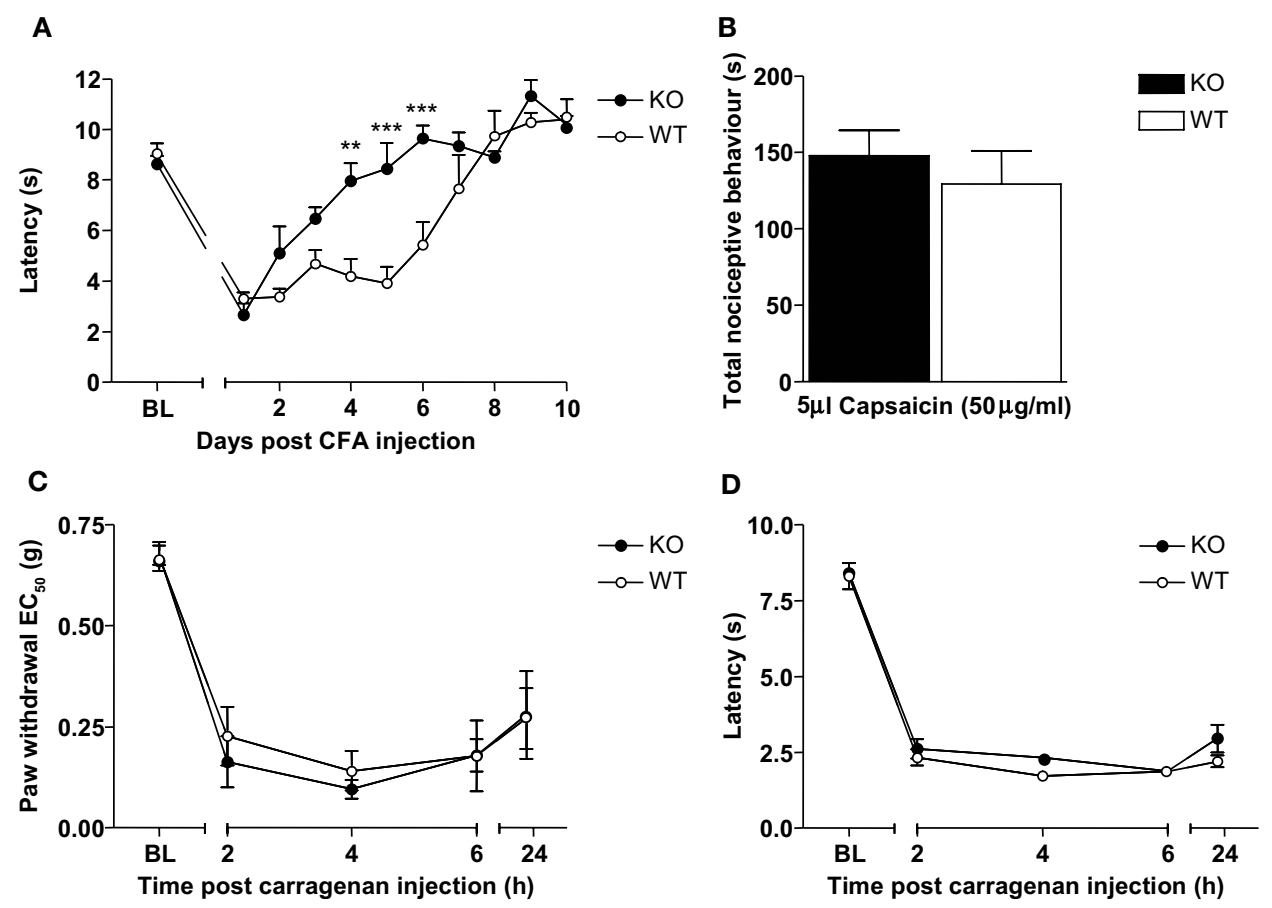

FIGURE 3 | GIra3-- mice display attenuated CFA behaviour, but show no differences in other inflammatory models. (A) Paw withdrawal latencies following unilateral intraplantar CFA (20 $\mu$ l) were significant versus contralateral responses for wild-type mice days $1-6$ and days $1-3$ for G/ra3 ${ }^{-}$mice $(p<0.001)$. Paw withdrawal latencies of G/ra3 ${ }^{-1}$ mice were significantly different from wildtype mice at days $4\left(p<0.01,{ }^{* *}\right), 5$ and $6\left(p<0.001,{ }^{* * *}\right)\left(G / r a 3^{-1}, n=7\right.$; wild type, $n=5$ ). (B) Total nociceptive behaviour following unilateral intraplantar capsaicin $(5 \mu \mathrm{l}, 50 \mu \mathrm{g} / \mathrm{ml})$ was not significantly different between genotypes (Glra3 ${ }^{-}, n=12$; wild type, $n=12$ ). (C) Significant mechanical hypersensitivity and (D) thermal hypersensitivity was observed at all timepoints following unilateral intraplantar carrageenan $(20 \mu \mathrm{l}, 2 \% \mathrm{w} / \mathrm{v})$ when compared with contralateral controls $(p<0.05-p<0.001)$ but indistinguishable between genotypes (Glra3-1-, $n=5$; wild type, $n=7$ ). Data are presented as mean \pm SEM and analysed using two-way repeated measures ANOVA, Bonferroni post hoc analysis.

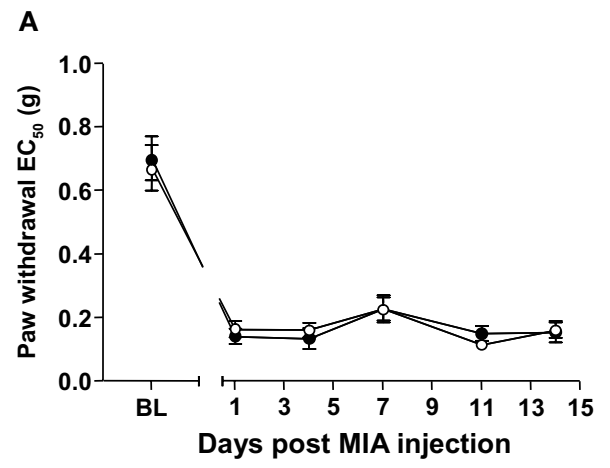

FIGURE 4 | Glra3 ${ }^{-1}$ mice show no differences in behavioural hypersensitivity in models of osteoarthritis or rheumatoid arthritis. (A) Significant mechanical hypersensitivity was observed at all timepoints following unilateral intra-articular MIA $(10 \mu \mathrm{l}, 5 \mathrm{mg} / \mathrm{ml})$ in both genotypes when compared with contralateral controls $(n=6 ; p<0.001)$. (B) Significant thermal
B

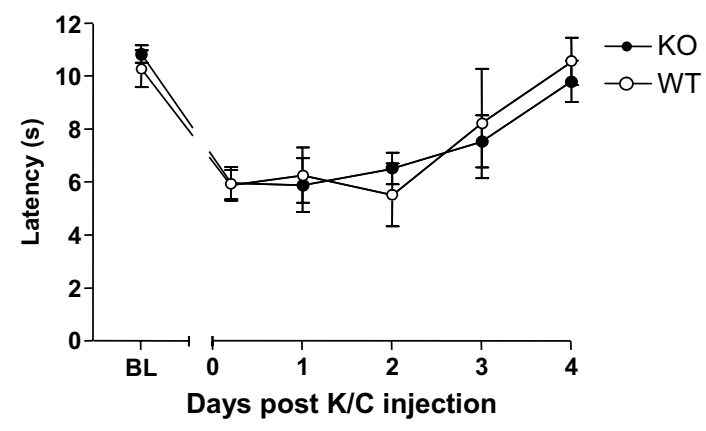

hypersensitivity was observed at days 1 and 2 following unilateral intra-articular $\mathrm{K} / \mathrm{C}$ injection $(15 \mu \mathrm{l}, 3 \% \mathrm{w} / \mathrm{v})$ in both genotypes when compared with contralateral controls ( $n=6 ; p<0.05$ ). No differences were observed between genotypes. Data are presented as mean \pm SEM and analysed using two-way repeated measures ANOVA, Bonferroni post hoc analysis.

\section{NEUROPATHIC PAIN BEHAVIOUR}

Following the induction of peripheral nerve injury using the partial sciatic nerve ligation model, both $\mathrm{Glra}^{-1-}$ and wild-type littermates developed robust and persistent mechanical and thermal hypersensitivities from the onset of testing (Figures 5A,B). Significant mechanical hypersensitivity was observed in $\mathrm{Glra3}^{-/-}$mice at days
3, 5, 7 and $14(p<0.01-p<0.001)$ and wild-type mice at days 3-14 $(p<0.05-p<0.01)$ following surgery compared with contralateral controls $(n=6)$. Significant thermal hypersensitivity was observed in $\mathrm{Glra3}^{-1-}$ mice and wild-type mice at all days following surgery $(p<0.001)$ compared with contralateral controls $(n=6)$. Statistical analysis revealed no significant difference in $\mathrm{Glra3}^{-1-}$ mice 


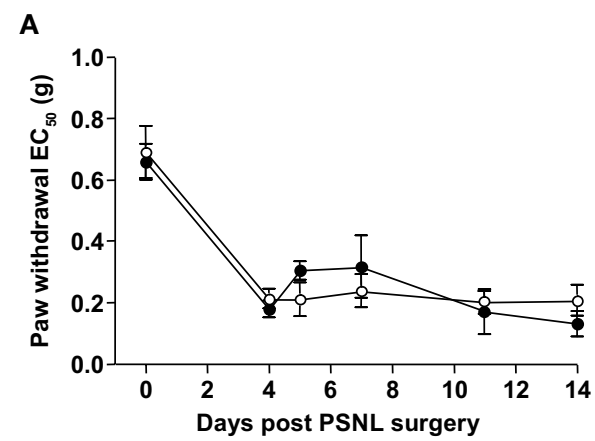

FIGURE 5 | GIra3-- mice show no differences in behavioural hypersensitivitiy following experimentally induced peripheral neuropathy. (A) Significant mechanical hypersensitivity was observed in G/ra3 ${ }^{-/-}$mice at days $3,5,7$ and $14(p<0.01-p<0.001)$ and wild-type mice at days $3-14(p<0.05-$ $p<0.01)$ following surgery compared with contralateral controls $(n=6)$.

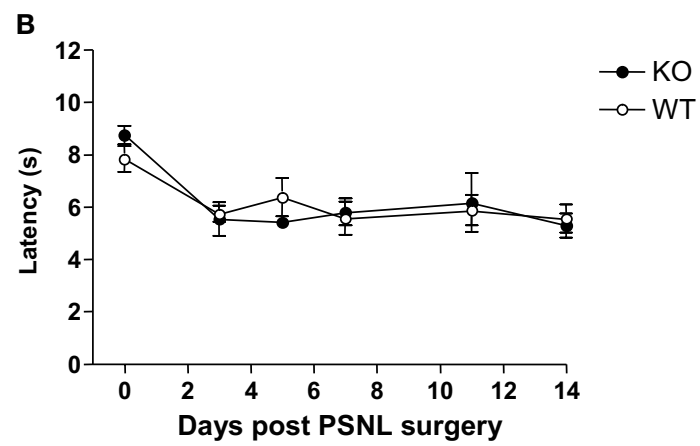

(B) Significant thermal hypersensitivity was observed in Glra3-- mice and wildtype mice at all days following surgery $(p<0.001)$ compared with contralateral controls $(n=6)$. No differences were observed between genotypes. Data are presented as mean \pm SEM and analysed using two-way repeated measures ANOVA, Bonferroni post hoc analysis. in either the magnitude or duration of thermal and mechanical hypersensitivities following the induction of nerve injury when compared with wild-type control littermates $(p>0.05)$.

\section{VISCERAL PAIN}

The visceromotor response (VMR), evoked by the distension of the colon by a balloon inserted intra-colonically, provides a quantifiable measure of visceral pain (visceral hyperalgesia). In rodents, pressures which elicit pain in humans evoke graded abdominal muscle contractions (measured as the VMR), tachycardia and the activation of primary afferent visceral nerves (e.g. pelvic nerve) and subsequent activation of second order projection neurones (Kamp et al., 2003). The response measured at $0 \mathrm{mmHg}$ (with the balloon deflated) was considered as the baseline response; $30 \mathrm{mmHg}$ was considered mildly noxious and $60 \mathrm{mmHg}$ as significantly noxious. Both wild-type and $\mathrm{Glra3}^{-/-}$ mice displayed coded responses to the graded stimuli (Figure 6), however, the responses were not significantly different between the groups $(p>0.05)$.

\section{DISCUSSION}

This study confirms, consistent with previous findings, that GlyR $\alpha 3$ has a significant role in certain inflammatory pain states. In this study, we have used knockout mice to investigate the role of GlyR $\alpha 3$ in a variety of acute, persistent and clinically relevant behavioural pain models as well as neuropathic and visceral pain models. Moreover, in vivo electrophysiology has been used to investigate the potential role of these channels in response to suprathreshold stimuli, which equate to intensities that produce high pain scores such as those seen in the clinic.

\section{ACUTE PAIN THRESHOLDS}

Consistent with previous findings, acute behavioural thermal and mechanical thresholds in $\mathrm{Glra3}^{-1-}$ mice were unaltered (Harvey et al., 2004). These behavioural findings are re-enforced by the electrophysiological characterisation of $\mathrm{Glra3}^{-1-}$ mice to an array of different modalities ranging from threshold to suprathreshold intensities, suggesting that GlyR $\alpha 3$ is not implicated in acute

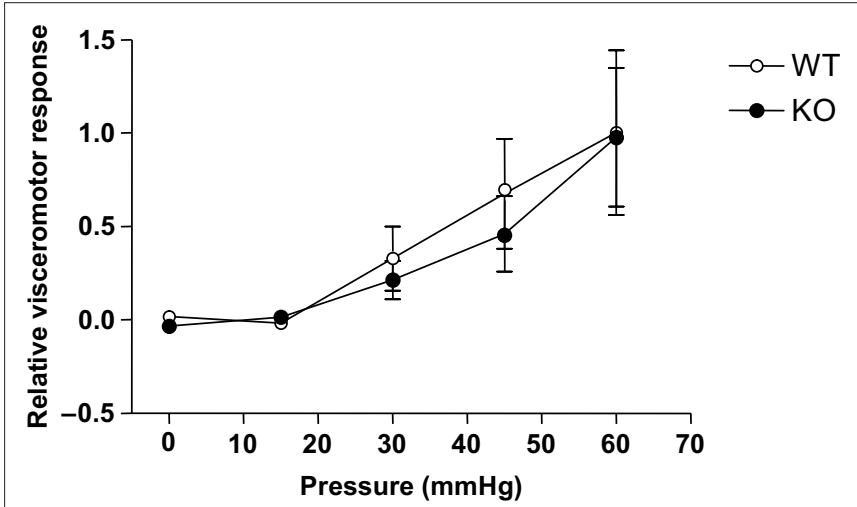

FIGURE 6 | GIra3-1- mice show no differences in visceromotor responses to colorectal distension. Isoflurane anesthetised mice display graded visceromotor responses to increasing colorectal pressures. No differences were observed between genotypes (G/ra3 ${ }^{-1}, n=5$; wild type, $n=7$ ). Data are presented as mean (normalised to maximum wild-type control) \pm SEM.

nociception. These characteristics are advantageous when investigating potential therapeutic targets since ideal analgesic targets should be pain-state specific, leaving normal nociceptive processing and thus normal sensation unaltered.

\section{INFLAMMATORY PAIN MODELS}

Although the role of GlyR $\alpha 3$ in inflammatory pain has been previously investigated in selected models, this study expands on the current work by investigating its potential role in other inflammatory pain states including capsaicin and carrageenan inflammation and clinically relevant models such as the kaolin/carrageenan (K/C) model of rheumatoid arthritis and the monosodium iodoacetate model of osteoarthritis. CFA-induced behavioural hypersensitivity was reconfirmed in this study, where $\mathrm{Glra3}^{-1-}$ mice displayed an accelerated rate of recovery, in line with previous findings (Harvey et al., 2004). Since GlyR $\alpha 3$ displayed this inflammatory phenotype, its potential role in other models of inflammation was investigated. 
Intraplantar capsaicin elicits immediate spontaneous pain behaviour in rodents through the activation of the vanilloid receptor 1 (VR1/TRPV1). This evoked nocifensive behaviour is thought to arise from the direct activation of $\mathrm{C}$-fibres rather than as a consequence of local inflammation per se. Although capsaicin-induced C fibre activation has previously been found to elicit prostaglandin $\mathrm{E}_{2}$ release in spinal cord slices (Malmberg and Yaksh, 1994), the exact spinal mechanisms have not been fully established. In this study, no behavioural phenotype was observed following intraplantar capsaicin injection in $\mathrm{Glra3}^{-/-}$mice.

Intraplantar injection of carrageenan evokes both mechanical and thermal hypersensitivity in rodents. These behaviours can be subdivided into an acute phase (2-6h) and sub-acute phase (24 h) (Kolhekar et al., 1997). Prostaglandin synthesis occurs both at peripheral and spinal sites since both systemic and intrathecal cyclo-oxygenase (COX) inhibition attenuates nocifensive behaviour. However, intrathecal COX inhibitors were only effective when given prior to carrageenan injection (Dirig et al., 1998). A later study found that spinal CSF levels of $\mathrm{PGE}_{2}$ peaked in the acute phase but remained significantly elevated up to $24 \mathrm{~h}$ (Guay et al., 2004). Taken together, this suggests that there is potentially only a limited time frame where spinal $\mathrm{PGE}_{2}$ is contributing to the pain behaviours, whereas peripheral or supraspinal sites are involved in both the induction and maintenance of carrageenan-evoked pain behaviours. In this study, no phenotype was observed in either the acute or sub-acute phase of carrageenan behavioural hypersensitivity in $\mathrm{Glra3}^{-1-}$ mice. Therefore, although spinal prostaglandins are involved in the induction, and to a lesser extent the maintenance of this model, the $\mathrm{EP}_{2}$ receptor - GlyR $\alpha 3$ signalling pathway is unlikely to represent the downstream substrate.

\section{ARTHRITIC PAIN MODELS}

Arthritic pain, as a result of either rheumatoid arthritis or osteoarthritis, represents a major chronic healthcare problem escalating with our aging and obese population (Neugebauer et al., 2007). Although different models exist for their study, the chemical models of MIA, for osteoarthritis, and K/C for rheumatoid arthritis, are popular among pain researchers. In this study, mice displayed significant behavioural hypersensitivity in the paw of the affected limb in both arthritic models, consistent with other literature (Yang et al., 1996; Harvey and Dickenson, 2009). Similar to other peripheral inflammatory models, spinal prostanoids are thought to contribute largely to the observed pain behaviours. Infrapatellar $\mathrm{K} / \mathrm{C}$ injection leads to a robust and persistent (up to $72 \mathrm{~h}$ ) increase in spinal $\mathrm{PGE}_{2}$ levels in rats (Dirig and Yaksh, 1999). In the MIA model of osteoarthritis, NSAIDs are only effective at the early stages of the model, suggesting a switch in the underlying mechanisms at later time points although these have not yet been fully established (Bove et al., 2003; Pomonis et al., 2005; Vonsy et al., 2008). In this study, it would appear that despite good evidence for central prostanoid release, GlyR $\alpha 3$ is not implicated in the underlying pain pathophysiology.

\section{NEUROPATHIC PAIN}

The benefits of using NSAIDs to treat neuropathic pain remains controversial, and at best they may only be effective at very early stages following the nerve insult (Ma et al., 2002; Schafers et al.,
2004). In this study, analysis of the pain behaviours following the induction of peripheral nerve injury using the partial sciatic nerve injury model revealed that $\mathrm{Glra}^{-/-}$mice developed profound mechanical and thermal hypersensitivities indistinguishable from wild-type littermates. This is in accordance with previous studies investigating the role of GlyR $\alpha 3$ in the chronic constriction injury model of neuropathic pain (Racz et al., 2005; Hösl et al., 2006). It is apparent, therefore, that unlike its clear role in certain types of inflammatory pain, GlyR $\alpha 3$ is not critical for the induction or maintenance of neuropathic pain.

\section{VISCERAL PAIN}

Bowel disorders such as irritable bowel syndrome (IBS) are characterised by poorly localised abdominal pain and sufferers display visceral hypersensitivity to experimental colorectal distension compared with healthy volunteers (Verne et al., 2001). To investigate the potential role of GlyR $\alpha 3$ in such bowel complaints we used a method of colorectal distension which produces a quantifiable visceromotor response in lightly anaesthetised mice. In this study, although the magnitude of the VMR was dependent on the applied pressure, no significant differences were observed between genotypes. This contrasts with other studies using the acetic acid writhing test, which reported a significant attenuation in the writhing responses in $\mathrm{Glra3}^{-/-}$mice (Racz et al., 2005). This model, however, is often difficult to interpret as little is known about the pathophysiology following acetic acid injection. The acute testing regimen utilised in this study, however, may not be sufficiently long to involve the proposed $\mathrm{EP}_{2}$ receptor - GlyR $\alpha 3$ signalling pathway. A phenotype using the CRD model might be uncovered by future experiments involving colonic hypersensitivity where VMR are recorded in response to an irritant injected intra-colonically which enhance the VMR to both noxious and non-noxious intensities in rats (Coutinho et al., 1996), although care must be taken to avoid general systemic effects (Deitch et al., 1992).

\section{CONCLUSION}

Since the contribution of a particular molecular substrate to the nociceptive phenotype will most likely depend on the origin of the pain (e.g. inflammatory, neuropathic or dysfunctional), the temporal characteristics (i.e. acute or chronic), and the underlying sensitivity (peripheral or central) it is likely that different substrates underlie different pain states.

A selective role of GlyR $\alpha 3$ can be observed based on clear effects in the CFA model, yet there is no clear role in other inflammatory, neuropathic or visceral pain models. This study, therefore, suggests that GlyR $\alpha 3$ may play an important role in mediating $\mathrm{PGE}_{2}-$ induced sensitisation, but only in certain pain states. Previously, GlyR $\alpha 3$ has been found not to contribute to behavioural hypersensitivity following formalin injection (Hösl et al., 2006). Although this model, like most models of peripheral injury, evokes spinal $\mathrm{PGE}_{2}$ release and is attenuated by COX inhibitors, it most likely reflects a different downstream target since EP2 receptor knockout mice, but not $\mathrm{Glra}^{-1-}$ mice, displayed attenuated nocifensive behaviour. This could also hold true for the other inflammatory models investigated in this study and may point to the differential involvement of other prostanoids and EP receptors, located either 
centrally or peripherally, that may activate different molecular effectors (e.g. Matsumoto et al., 2005; Hutchinson et al., 2009). What is clear, however, is that different inflammatory pain states are underpinned by different molecular substrates such that their investigation will help to dissect out pain-state specific mechanisms. These observations may be reflected in different clinical phenotypes and, as such, a more mechanistic based approach may be required in the clinic.

\section{REFERENCES}

Ahmadi, S., Lippross, S., Neuhuber, W. L., and Zeilhofer, H. U. (2002). PGE selectively blocks inhibitory glycinergic neurotransmission onto rat superficial dorsal horn neurons. Nat. Neurosci. 5, 34-40.

Bove, S.E., Calcaterra, S.L., Brooker, R. M., Huber, C. M., Guzman, R. E., Juneau, P. L., Schrier, D. J., and Kilgore, K.S. (2003). Weight bearing as a measure of disease progression and efficacy of anti-inflammatory compounds in a model of monosodium iodoacetate-induced osteoarthritis. Osteoarthr. Cartil. 11, 821-830.

Chaplan, S. R., Bach, F. W., Pogrel, J. W., Chung, J. M., and Yaksh, T. L. (1994). Quantitative assessment of tactile allodynia in the rat paw. J. Neurosci. Methods 53, 55-63.

Coutinho, S. V., Meller, S. T., and Gebhart, G. F. (1996). Intracolonic zymosan produces visceral hyperalgesia in the rat that is mediated by spinal NMDA and non-NMDA receptors. Brain Res. 736, 7-15.

Deitch,E.A.,Specian, R.D., Grisham, M. B., and Berg, R. D. (1992). Zymosaninduced bacterial translocation: a study of mechanisms. Crit. Care Med. 20, 782-788.

Dirig, D. M., Isakson, P.C., and Yaksh, T. L. (1998). Effect of COX-1 and COX-2 inhibition on induction and maintenance of carrageenan-evoked thermal hyperalgesia in rats. J. Pharmacol. Exp. Ther. 285, 1031-1038.

Dirig, D. M., and Yaksh, T. L. (1999). In vitro prostanoid release from spinal cord following peripheral inflammation: effects of substance P, NMDA and capsaicin. Br. J. Pharmacol. 126, 1333-1340.

Guay, J., Bateman, K., Gordon, R., Mancini, J., and Riendeau, D. (2004). Carrageenan-induced paw edema in rat elicits a predominant prostaglandin $\mathrm{E} 2\left(\mathrm{PGE}_{2}\right)$ response in the central nervous system associated with the induction of microsomal $\mathrm{PGE}_{2}$ synthase-1. J. Biol. Chem. 279, 24866-24872.

Harvey, R. J., Depner, U. B., Wässle, H., Ahmadi, S., Heindl, C., Reinold, H., Smart, T. G., Harvey, K., Schutz, B., Abo-Salem, O. M., Zimmer, A., Poisbeau, P., Welzl, H., Wolfer, D. P., Betz, H., Zeilhofer, H. U., and Müller, U. (2004). GlyR $\alpha 3$ : an essential target for spinal $\mathrm{PGE}_{2}$-mediated inflammatory pain sensitization. Science 304, 884-887.

Harvey, V.L., and Dickenson, A.H. (2009). Behavioural and electrophysiological characterisation of experimentally induced osteoarthritis and neuropathy in C57Bl/6 mice. Mol. Pain 5, 18.

Hösl, K., Reinold, H., Harvey, R. J., Müller, U., Narumiya, S., and Zeilhofer, H.U. (2006). Spinal prostaglandin $\mathrm{E}$ receptors of the EP2 subtype and the glycine receptor $\alpha 3$ subunit, which mediate central inflammatory hyperalgesia, do not contribute to pain after peripheral nerve injury or formalin injection. Pain 126, 46-53.

Hutchinson, A. J., Chou, C. L., Israel, D. D., Xu, W., and Regan, J. W. (2009). Activation of EP2 prostanoid receptors in human glial cell lines stimulates the secretion of BDNF. Neurochem. Int. 54, 439-446. Tillman, S. R., and Gebhart, G. F. (2003). Quantitative assessment and characterization of visceral nociception and hyperalgesia in mice. Am. J. Physiol. Gastrointest. Liver Physiol. 284, G434-G444. (1997). Thalamic NMDA receptors modulate inflammation-produced hyperalgesia in the rat. Pain 71,31-40.
Kamp, E. H., Jones, R. C., 3rd,

Kolhekar, R., Murphy, S., and Gebhart, G. F.

\section{ACKNOWLEDGMENTS}

Robert J. Harvey and Anthony H. Dickenson conceived the study, Victoria L. Harvey and Anthony H. Dickenson designed and performed the experiments, analysed data and wrote the manuscript with input from all authors. Alex Caley provided mouse genotyping; Robert J. Harvey and Ulrike C. Müller generated and supplied the Glra3 ${ }^{-1-}$ mice. This work was supported by MRC Project Grant (G0500833) to Robert J. Harvey and Anthony H. Dickenson.

Ma, W., Du, W., and Eisenach, J.C. (2002). Role for both spinal cord COX-1 and COX-2 in maintenance of mechanical hypersensitivity following peripheral nerve injury. Brain Res. 937, 94-99.

Malmberg, A. B., and Basbaum, A. I. (1998). Partial sciatic nerve injury in the mouse as a model of neuropathic pain: behavioral and neuroanatomical correlates. Pain 76, 215-222.

Malmberg, A. B., and Yaksh, T. L. (1994) Capsaicin-evoked prostaglandin E2 release in spinal cord slices: relative effect of cyclooxygenase inhibitors. Eur. J. Pharmacol. 271:293-299.

Matsumoto, S., Ikeda, M., Yoshida, S. Tanimoto, T., Takeda, M., and Nasu, M. (2005). Prostaglandin E2-induced modification of tetrodotoxin-resistant $\mathrm{Na}^{+}$currents involves activation of both EP2 and EP4 receptors in neonatal rat nodose ganglion neurones. Br. J. Pharmacol. 145, 503-513.

Neugebauer, V., Han, J. S., Adwanikar, H. Fu, Y., and Ji, G. (2007). Techniques for assessing knee joint pain in arthritis. Mol. Pain 3, 8.

Pomonis, J.D., Boulet, J.M., Gottshall, S. L., Phillips, S., Sellers, R., Bunton, T., and Walker, K. (2005). Development and pharmacological characterization of a rat model of osteoarthritis pain. Pain 114, 339-346.

Racz, I., Schutz, B., Abo-Salem, O. M. and Zimmer, A. (2005). Visceral, inflammatory and neuropathic pain in glycine receptor $\alpha 3$-deficient mice. Neuroreport 16, 2025-2028.

Reinold, H., Ahmadi, S., Depner, U. B. Layh, B., Heindl, C., Hamza, M., Pahl, A., Brune, K., Narumiya, S., Müller, U., and Zeilhofer, H. U. (2005). Spinal inflammatory hyperalgesia is mediated by prostaglandin E receptors of the EP2 subtype. J. Clin. Invest. 115, 673-679.

Schafers, M., Marziniak, M., Sorkin, L. S., Yaksh, T. L., and Sommer, C. (2004).
Cyclooxygenase inhibition in nerve-injury- and TNF-induced hyperalgesia in the rat. Exp. Neurol. 185, 160-168.

Verne, G. N., Robinson, M. E., and Price, D. D. (2001). Hypersensitivity to visceral and cutaneous pain in the irritable bowel syndrome. Pain 93, 7-14.

Vonsy, J. L., Ghandehari, J., and Dickenson, A. H. (2008). Differential analgesic effects of morphine and gabapentin on behavioural measures of pain and disability in a model of osteoarthritis pain in rats. Eur. J. Pain 13, 786-793.

Yang, L. C., Marsala, M., and Yaksh, T. L. (1996). Characterization of time course of spinal amino acids, citrulline and $\mathrm{PGE}_{2}$ release after carrageenan/ kaolin-induced knee joint inflammation: a chronic microdialysis study. Pain 67, 345-354.

Conflict of Interest Statement: The authors declare that the research was conducted in the absence of any commercial or financial relationship that could be construed as a potential conflict of interest.

Received: 31 July 2009; paper pending published: 16 August 2009; accepted: 27 August 2009; published online: 04 November 2009. Citation: Harvey VL, Caley A, Müller $U C$, Harvey RJ and Dickenson $A H$ (2009) A selective role for $\alpha 3$ subunit glycine receptors in inflammatory pain. Front. Mol. Neurosci. 2:14. doi: 10.3389/neuro.02.014.2009

Copyright (C) 2009 Harvey, Caley, Müller, Harvey and Dickenson. This is anopen-access article subject to an exclusive license agreement between the authors and the Frontiers Research Foundation, which permits unrestricted use, distribution, and reproduction in any medium, provided the original authors and source are credited. 